\title{
Spontaneous Intracranial Hypotension: A Systematic Imaging Approach for CSF Leak Localization and Management Based on MRI and Digital Subtraction Myelography
}

\author{
(D) R.I. Farb, DP.J. Nicholson, (DP.W. Peng, (DE.M. Massicotte, (D). Lay, DT. Krings, and (D).G. terBrugge
}

\begin{abstract}
BACKGROUND AND PURPOSE: Localization of the culprit CSF leak in patients with spontaneous intracranial hypotension can be difficult and is inconsistently achieved. We present a high yield systematic imaging strategy using brain and spine MRI combined with digital subtraction myelography for CSF leak localization.
\end{abstract}

MATERIALS AND METHODS: During a 2-year period, patients with spontaneous intracranial hypotension at our institution underwent MR imaging to determine the presence or absence of a spinal longitudinal extradural collection. Digital subtraction myelography was then performed in patients positive for spinal longitudinal extradural CSF collection primarily in the prone position and in patients negative for spinal longitudinal extradural CSF collection in the lateral decubitus positions.

RESULTS: Thirty-one consecutive patients with spontaneous intracranial hypotension were included. The site of CSF leakage was definitively located in 27 (87\%). Of these, 21 were positive for spinal longitudinal extradural CSF collection and categorized as having a ventral (type 1, fifteen [48\%]) or lateral dural tear (type 2; four [13\%]). Ten patients were negative for spinal longitudinal extradural CSF collection and were categorized as having a CSF-venous fistula (type 3, seven [23\%]) or distal nerve root sleeve leak (type 4, one [3\%]). The locations of leakage of 2 patients positive for spinal longitudinal extradural CSF collection remain undefined due to resolution of spontaneous intracranial hypotension before repeat digital subtraction myelography. In 2 (7\%) patients negative for spinal longitudinal extradural CSF collection, the site of leakage could not be localized. Nine of 21 (43\%) patients positive for spinal longitudinal extradural CSF collection were treated successfully with an epidural blood patch, and 12 required an operation. Of the 10 patients negative for spinal longitudinal extradural CSF collection (8 localized), none were effectively treated with an epidural blood patch, and all have undergone $(n=7)$ or are awaiting $(n=1)$ an operation.

CONCLUSIONS: Patients positive for spinal longitudinal extradural CSF collection are best positioned prone for digital subtraction myelography and may warrant additional attempts at a directed epidural blood patch. Patients negative for spinal longitudinal extradural CSF collection are best evaluated in the decubitus positions to reveal a CSF-venous fistula, common in this population. Patients with CSF-venous fistula may forgo further epidural blood patch treatment and go on to surgical repair.

ABBREVIATIONS: CVF = CSF-venous fistula; DSM = digital subtraction myelography; $\mathrm{EBP}=$ epidural blood patch; $\mathrm{SIH}=$ spontaneous intracranial hypotension; SLEC $=$ spinal longitudinal extradural CSF collection; SLEC-N = negative for spinal longitudinal extradural CSF collection; SLEC-P = positive for spinal longitudinal extradural CSF collection

$\mathbf{T}$ he syndrome of spontaneous intracranial hypotension $(\mathrm{SIH})$ is caused by leakage of CSF from the thecal sac within or along the spinal canal. ${ }^{1-4}$ The leakage of CSF causes variable symptoms,

Received January 6, 2019; accepted after revision February 16.

From the Department of Medical Imaging, Division of Neuroradiology (R.I.F., P.J.N., T.K., K.G.t.B.), Department of Anesthesiology (P.W.P.), Department of Surgery, and Division of Neurosurgery (E.M.M.), Toronto Western Hospital, Toronto, Ontario, Canada; and Department of Medicine and Division of Neurology, Women's College Hospital (C.L.) University of Toronto, Toronto, Ontario, Canada.

Please address correspondence to Richard I. Farb, MD, Division of Neuroradiology, Department of Medical Imaging, University of Toronto, University Health Network, Toronto Western Hospital, New East Wing 3MC-430, 399 Bathurst St, Toronto, ON, M5T 2S8 Canada; e-mail: richard.farb@uhn.ca

-- Indicates open access to non-subscribers at www.ajnr.org

http://dx.doi.org/10.3174/ajnr.A6016 the most characteristic of which is a positional headache and is commonly associated with typical intracranial MR imaging findings or stigmata. ${ }^{5-10}$ We have also noted, as have other authors, ${ }^{2,11-13}$ that while many patients with SIH present with a spinal longitudinal extradural CSF collection (SLEC), some do not. ${ }^{5}$ These SLECs have a typical appearance (Fig 1) and, in our experience, are seen exclusively in patients with SIH with dural mechanical tears along the thecal sac. These dural tears are most commonly seen ventrally. ${ }^{5}$ Conversely, patients with more laterally placed CSF leakage along a nerve root sleeve, from a CSFvenous fistula $(\mathrm{CVF})$ or from a distal nerve root sleeve tear, do not have SLECs. We report here our experience using a strategy predicated on the presence or absence of SLECs to determine the preferred positioning of subsequent digital subtraction myelography 

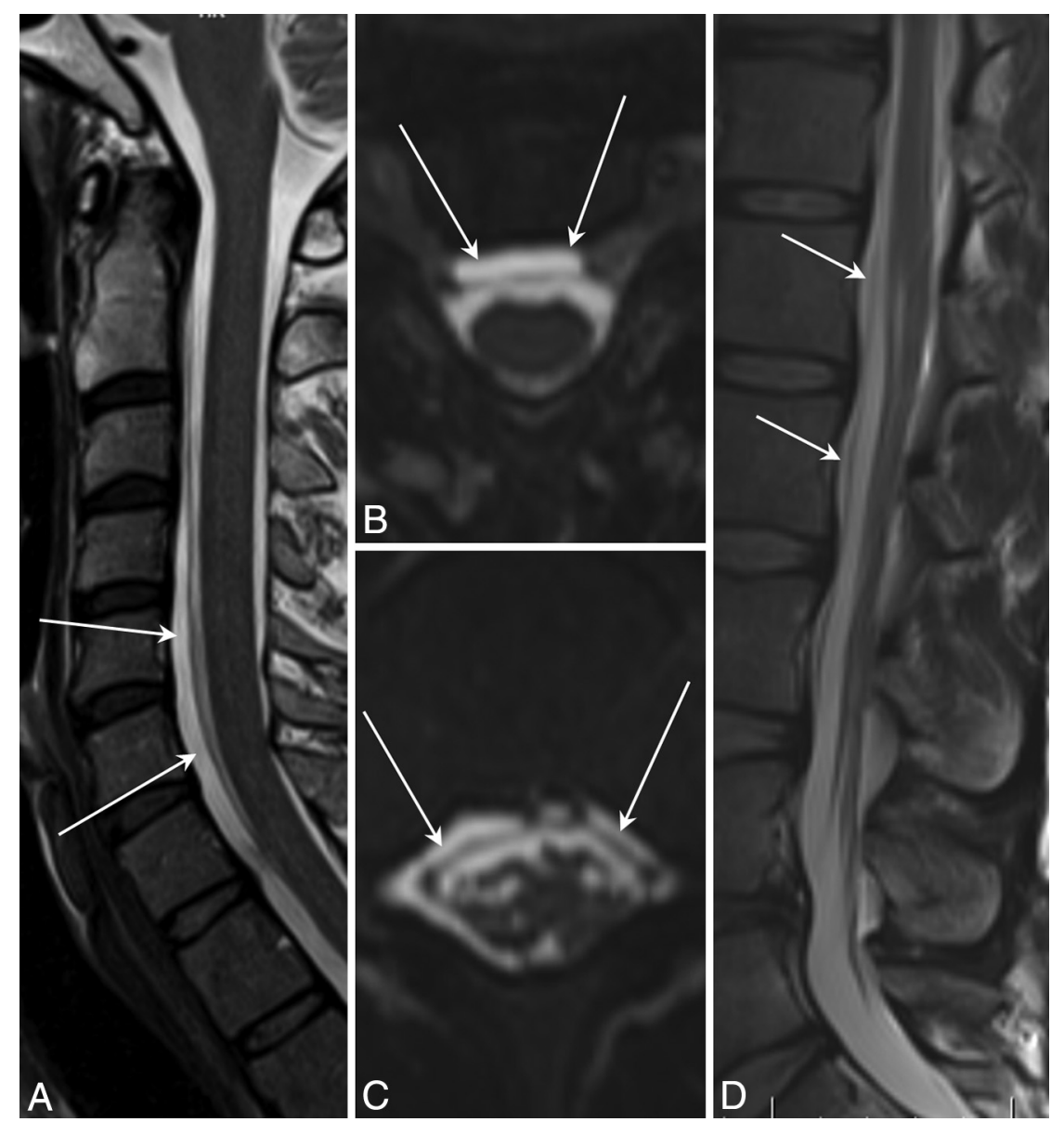

FIG 1. Spinal longitudinal extradural collections. A, Sagittal T2 FSE. B, Reformatted axial T2 SPACE images show SLECs (arrows) and displaced dura outlined by the CSF. $C$ and $D$, Images similar to $A$ and $B$ of the same patient show similar findings in the lower thoracic region.

Table 1: Prevalence of intracranial stigmata of hypotension

\begin{tabular}{lrrrrrr}
\hline & VDS & Sag & Hyg & SDH & Gad & Pit \\
\hline SLEC-P & & & & & & \\
$\quad$ No. of patients & 17 & 15 & 12 & 9 & 17 & 17 \\
\% & 81 & 71 & 57 & 43 & 81 & 81 \\
SLEC-N & & & & & & \\
$\quad$ No. of patients & 10 & 10 & 7 & 2 & 8 & 9 \\
$\%$ & 100 & 100 & 70 & 20 & 80 & 90 \\
\hline
\end{tabular}

Note:- Hyg indicates positive subdural hygromas over the convexity; Pit, pituitary engorgement; Sag, sagging appearance of the brain stem and posterior fossa structures; VDS, positive venous distension sign; Gad, gadolinium enhancement of the pachymeninges; SDH, patients who displayed subdural hemorrhage over the cerebral convexities.

(DSM). Specifically, patients positive for SLEC (SLEC-P) have CSF leaks that are best seen with DSM performed with the patient in the prone position, while those patients who are negative for SLEC (SLEC-N) have CSF leaks best seen with DSM performed with the patient in the lateral decubitus position. This imaging strategy was investigated for its ability to improve the yield for precisely locating the CSF leak and to help guide subsequent management.

\section{MATERIALS AND METHODS}

Institutional review board approval at University Health Network was obtained for this retrospective study.

The electronic patient record was reviewed for all patients who underwent MR imaging and DSM evaluation for SIH refractory to epidural blood patch (EBP) from December 2016 to October 2018. A patient was categorized as SLEC-P if the spinal MR imaging revealed the presence of an SLEC regardless of the intracranial findings. A patient was categorized as SLEC-N if intracranial findings were present without an SLEC on spinal MR imaging.

Patients in whom there was no evidence of intracranial MR imaging stigmata and in whom the spinal imaging was negative for an SLEC have not been consistently offered DSM at our institution and are not included in this report.

All patients positive for SLEC underwent DSM in the prone position. All patients negative for SLEC underwent DSM in the decubitus positions (first in the left lateral decubitus and then the right lateral decubitus position). DSMs were repeated in other remaining positions until a CSF leak was found, the patient's symptoms resolved or a set of 3 myelograms negative a leak was completed.

\section{MR Imaging}

All referred patients whose available imaging from outside institutions did not include routine brain and adequate whole-spine imaging within the previous 90 days underwent an MR imaging protocol at our institution, which included a 2-station (upper and lower) high-resolution sagittal T2 sampling perfection with application-optimized contrasts by using different flip angle evolution sequence (SPACE sequence; Siemens, Erlangen, Germany) (TE/ $\mathrm{TR}=125 / 900 \mathrm{~ms}$, flip angle $=150^{\circ}$, matrix $=317 \times 320$, FOV $=$ $360 \times 360 \mathrm{~mm}$, slice thickness $=0.9 \mathrm{~mm}$, echo-train length $=63$, $\mathrm{NEX}=1.4$, reformatted in the axial plane throughout the spine in 2-mm-thick intervals) to document the presence or absence of SLECs. A similarly positioned T1-weighted spin-echo sequence $\left(\mathrm{TE} / \mathrm{TR}=8.5 / 769 \mathrm{~ms}\right.$, flip angle $=150^{\circ}$, matrix $=320 \times 256$, FOV $=400 \times 400 \mathrm{~mm}$, slice thickness $=3.5 \mathrm{~mm}$, echo-train length $=4$, NEX $=1$ ) was also performed to help differentiate fluid from fat within the spinal canal. All images were reviewed by 1 neuroradiologist (R.I.F.) for the intracranial MR imaging stigmata of hypotension, including a sagging appearance of the brain and brain stem, venous distension, pituitary gland enlargement, pachymeningeal enhancement, subdural hygromas, and subdural hemorrhages (Table 1). ${ }^{6,7,14,15}$ The spinal images were specifically reviewed to identify the presence or absence of an SLEC.

\section{DSM}

All patients positive and negative for SLEC subsequently underwent DSM. All DSM examinations at our institution are currently performed by 1 neuroradiologist (R.I.F.). 
Methodology common to all DSM examinations in this study is similar to that previously described ${ }^{16}$ with modifications described below.

The DSMs at our institution were performed with local anesthetic without intravenous sedation. All DSM examinations were performed on a biplane neuroangiographic system. Due to limitations of the equipment along with patient positioning, imaging was always performed in a single plane using the larger $(40 \times 30$ $\mathrm{cm}$ ) intensifier. Iohexol (Omnipaque 300; GE Healthcare, Piscataway, New Jersey) was used for all DSMs. All patients were positioned head down with bolsters and an additional $4^{\circ}-8^{\circ}$ of tilt added by the table-tilt function. For the initial DSM, all patients positive for SLEC were positioned prone and all patients negative for SLEC were in the left lateral decubitus position (left side down). If this initial DSM was negative for a CSF leak, the patient returned in 1-2 weeks for another DSM in differing position.

A 22-ga spinal needle was inserted under fluoroscopic guidance via a direct posterior approach at the L3-4 level. For patients in the prone position, a CSF opening pressure measurement was not attempted because CSF rarely ascended into the hub of the needle. Moreover, a result obtained with patients in this position would clearly be erroneous. For patients in the lateral decubitus position, an accurate CSF opening pressure was recorded. The remainder of the DSM examination was similar for all patients regardless of position. In all patients, a $0.5-\mathrm{mL}$ test injection ensured an intradural position of the needle tip. Following this, 10 $\mathrm{mL}$ of preservative-free normal saline was injected slowly for 2-4 minutes to further dilute and send the test dose down the spinal canal. The saline injection also potentially provided slight pressurization of the intradural compartment to encourage leaking. The DSM runs were performed with breath-hold and hand injection of contrast at a rate of $1 \mathrm{~mL}$ per second. The contrast was immediately chased with another $5-10 \mathrm{~mL}$ of saline to push the contrast through the tubing and down the thecal sac.

Image acquisition was performed at 1 frame per second for a total of 15-25 seconds per run. Each DSM procedure was divided into 2 separate injections (runs) of approximately 6 and $3.5 \mathrm{~mL}$ consecutively. The first injection covered the upper FOV and included from C3-4 to the midthoracic region. If it was positive for the site of leakage, then repeat imaging with a smaller FOV was performed with the remaining $3.5 \mathrm{~mL}$ of contrast. If the first injection was negative for the site of leakage, the FOV of the second injection was shifted to the lower thoracic and lumbar spine. Further saline (chasing) was usually not required for this lower injection. The multiple alternating injections of contrast and saline were controlled using a system of labeled syringes, 3-way stopcocks, and tubing. Following the second injection, the needle was removed and (if a leak was found) the patient was taken to the CT suite where scanning was typically performed with the patient in the prone or decubitus position from $\mathrm{C} 2$ to $\mathrm{S} 3$.

\section{RESULTS}

We identified 31 consecutive patients with SIH refractory to an initial EBP who underwent MR imaging of the brain and spine as well as DSM at our institution as described above. An SLEC was seen in 21 (68\%) of these 31 patients, thus categorizing them as SLEC-P. Conversely, 10 (32\%) patients were categorized as SLEC-N.

\section{Patients Positive for SLEC}

Of the 21 patients positive for SLEC, 18 (86\%) also showed intracranial stigmata of SIH, and the remaining 3 (14\%) did not (Table 1).

All 21 patients positive for SLEC underwent DSM in the prone position, which identified the site of CSF leakage in 19 (90\%). DSM was initially negative for a leak in $3(19 \%)$ patients positive for SLEC. One of these patients remained symptomatic and SLEC-P and underwent 3 separate DSMs negative for a leak at our institution (prone, right and left decubitus) and eventually underwent myelography at another institution, which demonstrated a ventral dural tear in the cervical spine not evident on initial prone DSM at our institution (personal written communication of 09/24/2018 with Dr Wouter Schievink, Department of Neurosurgery Cedars-Sinai Medical Center, Los Angeles, California). The remaining 2 patients positive for SLEC in whom the initial DSM was negative for a leak did not undergo further imaging because their symptoms resolved before repeat DSM (likely relating to an earlier EBP). In 3 patients positive for SLEC, the initial prone DSM was positive for a leak, which was better visualized with a subsequent repeat DSM (2 cases with repeat magnified view over the suspected ROI and in 1 case in which a decubitus positioning was optimal).

Thus, in 19 of the 21 patients positive for SLEC, a culprit dural tear was definitively identified. A typically appearing ventral hole within the dura related to degenerative disc disease was found in 15 of these 19 patients who were SLEC-P with DSM positive for a CSF leak. This type of CSF leak has been recognized and described by multiple authors, and we refer to this as a type 1 leak (Fig 2). A more lateral proximal nerve root sleeve tear unrelated to degenerative disc disease, which we have termed a type 2 leak (Fig 3), was found in the remaining 4 patients who were SLEC-P with DSM positive for a CSF leak.

A total of 27 DSMs were performed in the 21 patients positive for SLEC.

\section{Patients Negative for SLEC}

In 10 of the 31 consecutive patients (32\%) evaluated for persistent symptoms of SIH, there was no evidence of SLEC on spinal MR imaging. These patients all demonstrated typical intracranial stigmata of intracranial hypotension to varying degrees.

In 8 of the 10 patients negative for SLEC, lateral decubitus DSM identified a definitive CSF leakage site. These consisted of 7 cases of CVF, which we have termed as a type 3 leak (Fig 4) and 1 case of a distally extravasating nerve root sleeve leak, type 4 (Fig 5). In 2 of the patients negative for SLEC, a CSF leak could not be definitively identified despite the patient having completed the set of 3 complete DSMs. In total, 22 DSMs were performed in these 10 patients.

A total of 49 DSM examinations were performed in 31 patients.

The distribution of all localized CSF leaks is shown in Fig 6.

Aside from occasional mild transient headache, all patients tolerated the DSM procedures well without complications.

\section{CT}

CT was performed approximately 10-30 minutes following DSM in patients positive and negative for SLEC. The CT scans proved 


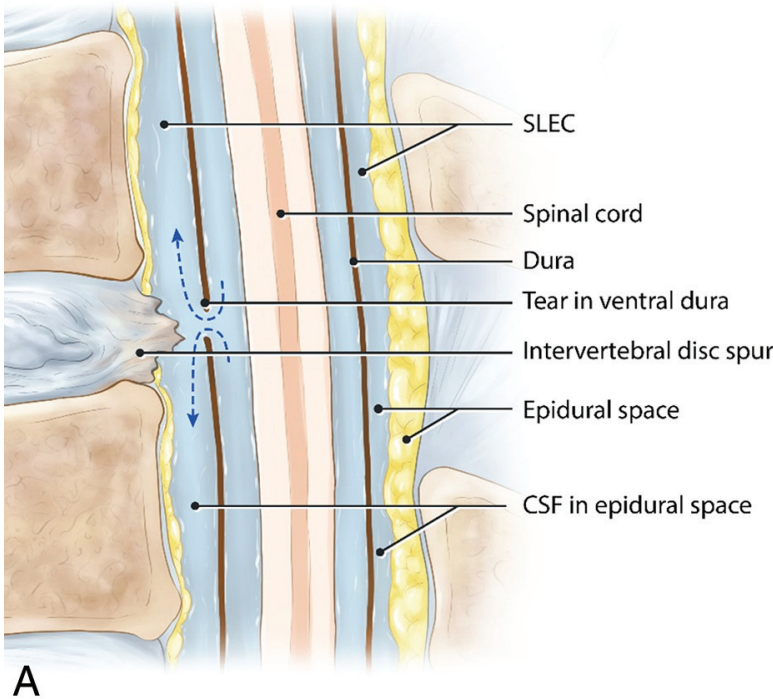

FIG 2. Type ICSF leak (SLEC-P). A, Schematic drawing shows the relationship of the intervertebral disc spur and a ventral dural tear. $B$, "Shoot though" lateral subtracted image of the thoracic spine DSM with the patient positioned prone on the table. The patient's head is toward the top of the image and feet at the bottom. The contrast material can be seen escaping from the ventral aspect of the thecal sac at the T7-8 level (arrow).

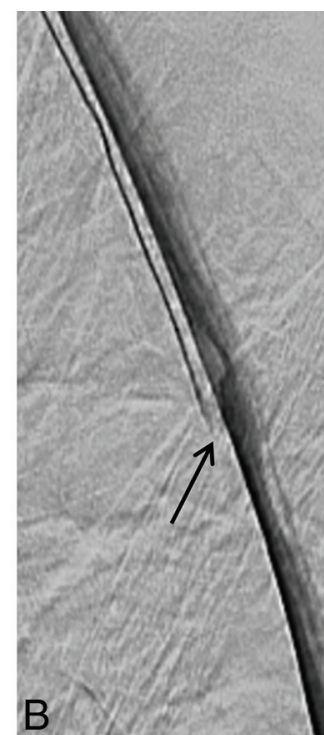

helpful for counting vertebral segments; however, they did not contribute further to the localization of the CSF leak. In patients positive for SLEC, the site of the dural tear demonstrated on the previous DSM could not be demonstrated on CT; however, the continued SLEC was usually evident. In patients negative for SLEC, scanning in this delayed fashion did not reveal evidence of the CVF even when reviewed with knowledge of the location of a CVF seen on the recent DSM.

\section{Intracranial Stigmata}

The most common intracranial stigmata were findings of a "positive venous distension sign", ${ }^{6}$ pachymeningeal enhancement on spin-echo postgadolinium imaging, and pituitary enlargement (Table 1). Three patients positive for SLEC demonstrated no intracranial stigmata on MR imaging. Nonetheless, these patients still
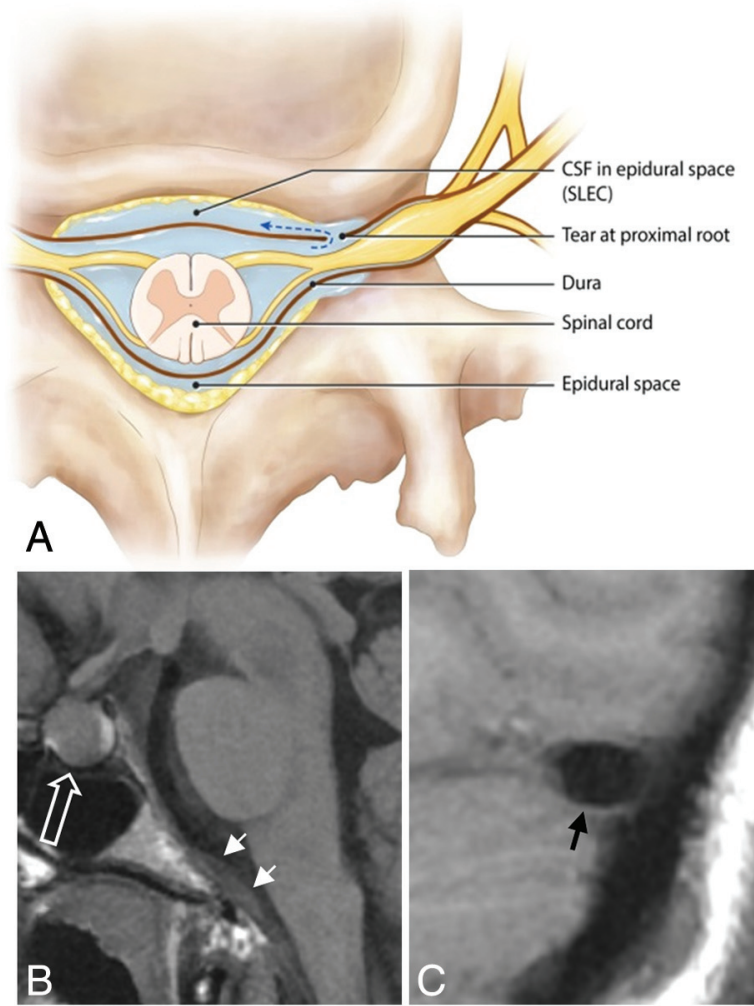
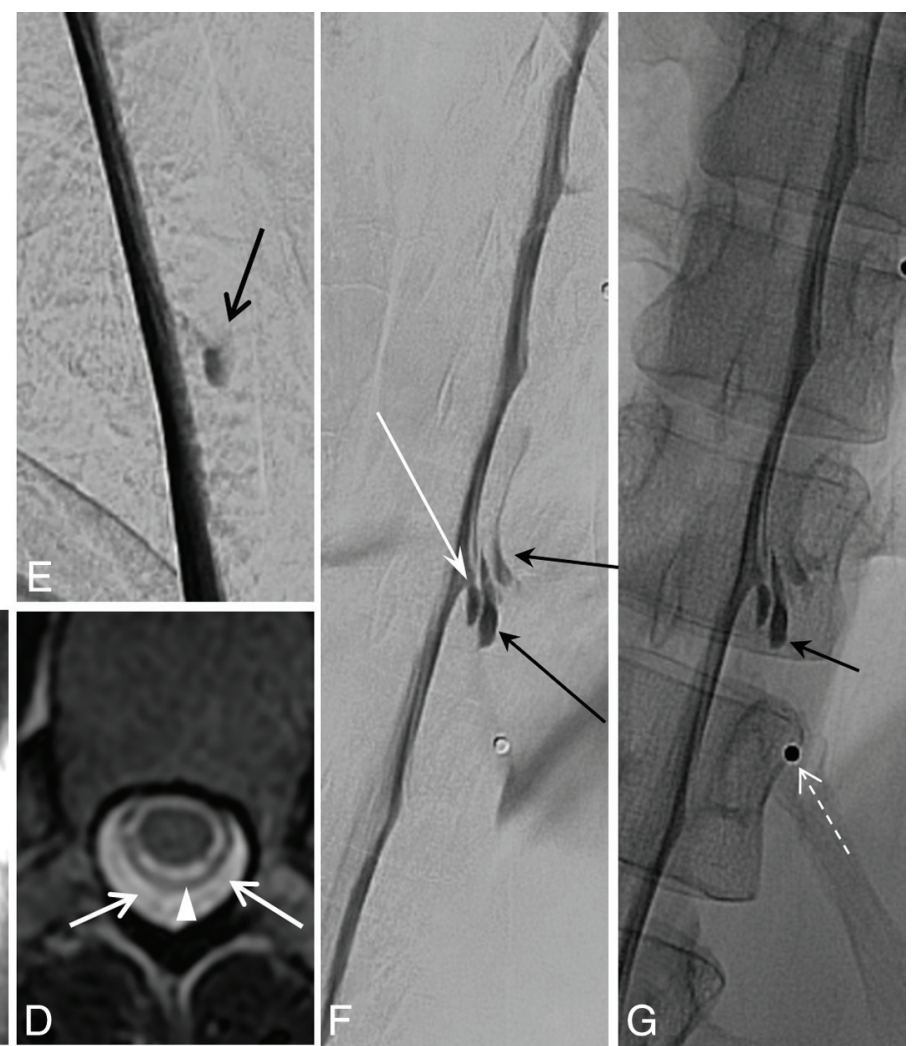

FIG 3. Type 2 CSF leak (SLEC-P). A, Schematic depiction of a proximal nerve root sleeve tear bridging the epidural and neural foraminal compartments. $B-G$, From a single patient. $B$, Sagittal TIWI of the brain shows the engorged pituitary gland (open white arrow) and dural thickening on the clivus (short white arrows). C, Sagittal T1WI of the brain shows a "positive venous distension sign" with a convex undersurface of the middle third of the dominant transverse sinus (short black arrow). D, T2-weighted axial MR image of the thoracic spine shows SLECs (white arrows) external to the dura (white arrowhead). E, Subtracted image from a prone thoracic DSM shows a posterolateral collection of contrast (black arrow). F and G, Subtracted and nonsubtracted images from a repeat right lateral decubitus DSM show contrast leaking into the extradural space (black arrows) from a tear along the proximal aspect of the right T11 root sleeve (long white arrow). Note the BB (nipple marker) placed on the skin for landmarking (dashed white arrow). 

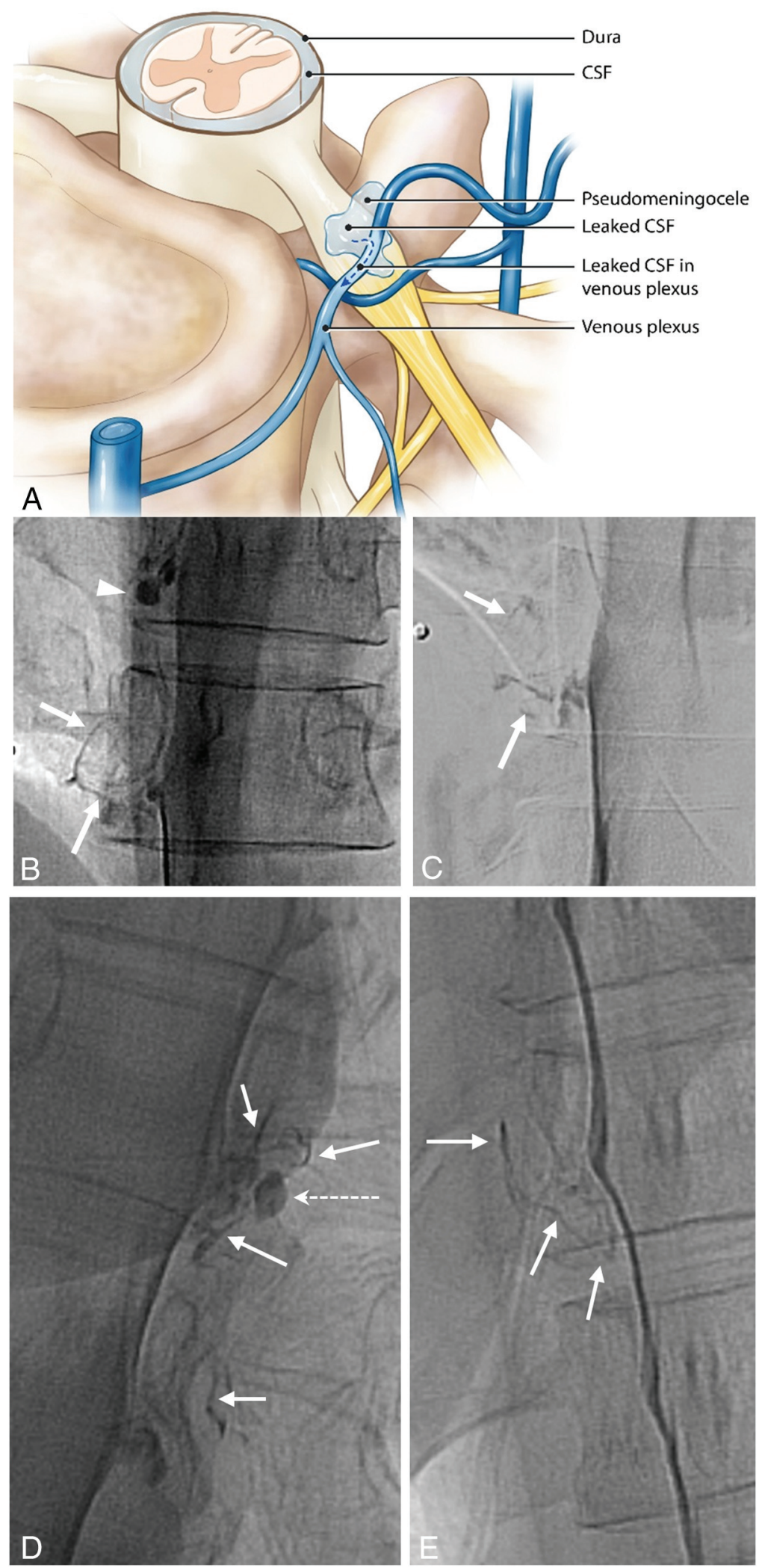

FIG 4. Type 3 CSF leak (SLEC-N). A, Schematic depiction of a CSF-to-venous fistula arising from a dural tear along the nerve root sleeve beyond the epidural compartment (see text). Nonsubtracted $(B)$ and magnified, subtracted $(C)$ images from separate left-side-down DSM runs in a patient negative for SLEC with SIH. A small vascular structure, in keeping with a tortuous vein of a CVF, can be seen coursing away from the root sleeve (arrows). An incidental normal diverticulum is also noted at the level above (arrowhead). $D$ and $E$, Nonsubtracted images of decubitus DSMs of 2 other similarly presenting patients negative for SLEC demonstrating CVFs. Globular collections of contrast (dashed arrow) are commonly seen near the expected zone of origin of the vein, possibly representing a focal extravasation (pseudomeningocele) of contrast or a diverticulum from which the vein appears to arise. 

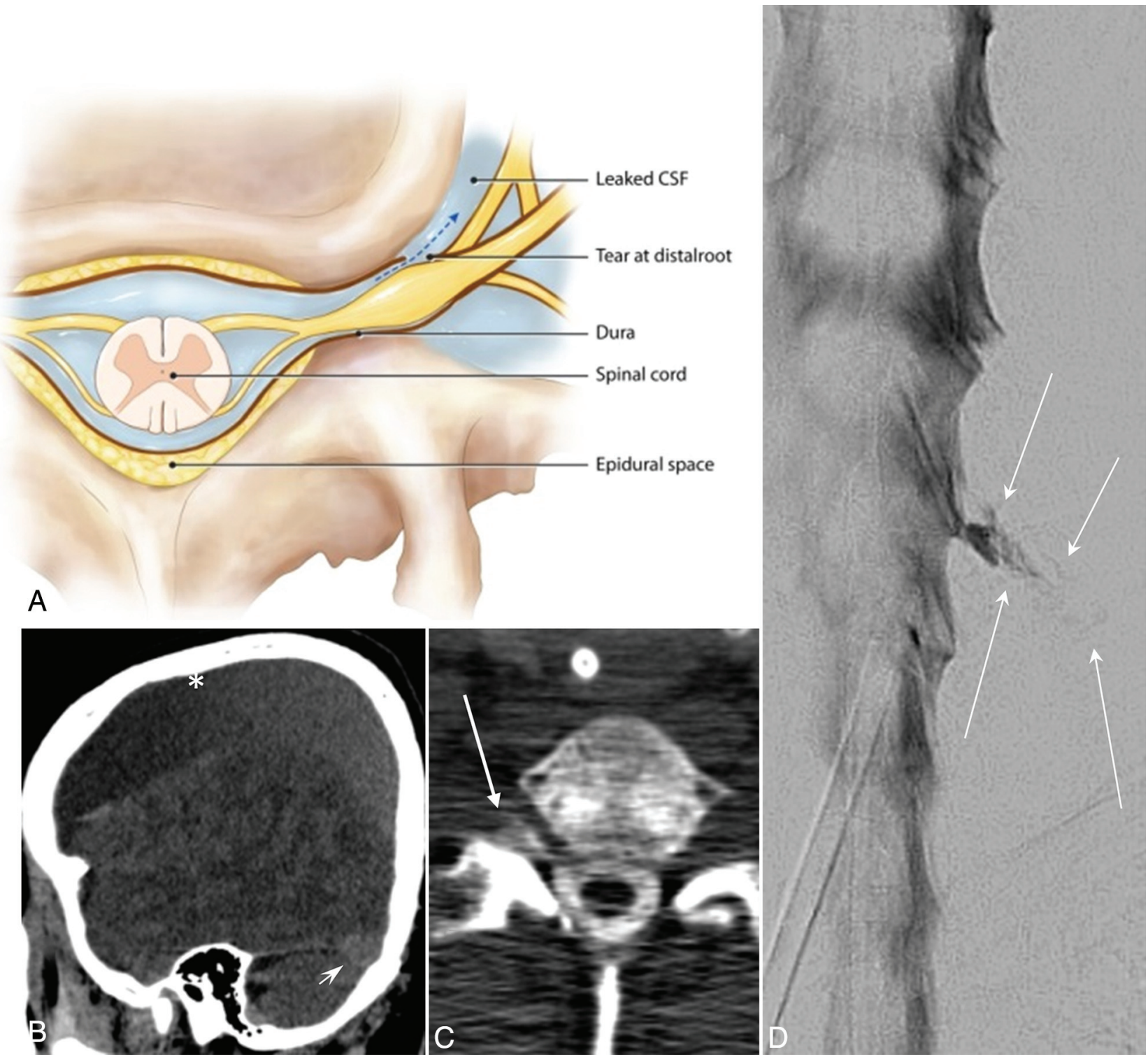

FIG 5. Type 4 CSF leak (SLEC-N). A, Schematic depiction of a distal nerve root sleeve dural tear occurring beyond the epidural compartment extravasating into the surrounding fascial planes and loose connective tissue without loculation or fistulization. $B$, CT of the head. Sagittal reformat in a patient negative for SLEC demonstrates large low-density (bilateral) subdural hemorrhages (asterisk). Note the prominent "venous distension sign" (short arrow) despite the large subdural hemorrhages. C, Axial CT image obtained 10-20 minutes post-DSM shows subtle extravasated contrast in the region of the right C8 nerve (arrow). Note that on this nondynamic CT (slightly degraded due to beam-hardening artifacts associated with the shoulders), there is little to help distinguish this extravasated contrast from a normal diverticulum. $D$, Subtracted image from a right-side-down decubitus DSM shows extravasation of contrast (arrows) into the paraspinal tissues from a leak along the mid-to-distal right C8 nerve root sleeve.

underwent DSM because of their compelling SLECs. A dural tear with an active CSF leak was identified in all 3 patients.

\section{Summary of Management}

Of the 31 patients in this series who underwent DSM for SIH, 27 $(87 \%)$ had their site of CSF leak definitively localized and 26 of these patients have undergone an operation or directed EBP for effective treatment, with complete or near-complete cessation of SIH symptoms (Table 2). One patient is awaiting surgery for an identified CVF at the time of this report. Two of the 31 patients were effectively treated with nondirected EBP (both were SLEC-P with the initial prone DSM negative for a CSF leak). Thus 29 (93\%) of the 31 consecutive patients with SIH were effectively managed with the strategy presented here. However, in 2 patients negative for SLEC, the CSF leak could not be localized despite exhaustive DSM; these patients continue with signs and symptoms of SIH. Follow-up MR imaging was available in 22 of the treated patients, and each showed complete resolution of all MR imaging findings. Four patients continue with some symptoms following treatment, 2 with improved-but-mild headache (beyond typical posttreatment "rebound hypertension") and 2 with mild back pain. Most interesting, these 4 patients have undergone follow-up MR showing complete resolution of stigmata.

In patients positive for SLEC, EBP was successful in treating $27 \%$ and $75 \%$ of patients with type 1 and 2 leaks, respectively. In 2 patients positive for SLEC, the type of leak was not defined at 


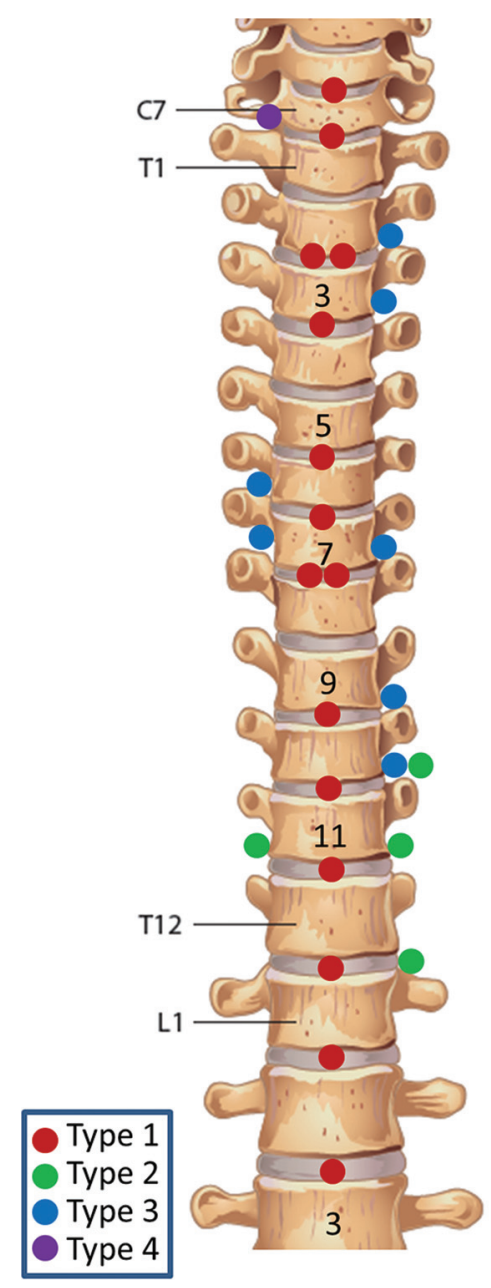

FIG 6. Distribution of CSF leaks.

DSM because it had likely already been slowed or closed by a prior EBP. Thus 9 of 21 (43\%) patients positive for SLEC were effectively treated with an EBP alone (usually directed to a region of the spine by leak localization). None of the 10 patients negative for SLEC (8 localized) were effectively treated with an EBP of any kind, and all have undergone or are awaiting surgical repair.

Our limited experience with CT-guided focal fibrin patching of CSF leaks does not parallel that reported by other authors. Following leak localization, CT-guided fibrin injection was performed in 4 patients with a type 1 leak, in 2 patients with a type 2 leak, and in 3 patients with a type 3 leak. In most cases, the fibrin injection failed within 48 hours. One patient with CVF reported some beneficial effect up to 6 months; however, this success was not borne out on follow-up MR imaging and the patient was referred for surgical repair, which resulted in clearing of all MR imaging signs of SIH. Fibrin patching failed to provide durable relief in all 9 injected patients.

\section{DISCUSSION}

Until recently, the cause of SIH had been unclear, and several etiologies had been postulated. ${ }^{10}$ With evidence presented here as well as that provided by other authors, ${ }^{1,2,5,11,17-19}$ it now appears that these leaks are predominantly, if not exclusively, due to mechanical tears, CVFs, and leaking nerve root sleeves. For guidance of management and efficiency of discussion, we have categorized the CSF leaks we have encountered into 4 types based on the morphology and distance from the midline. This is similar to a classification scheme previously proposed by Schievink et al. ${ }^{5}$

Type 1 is caused by degenerative disc disease creating a mechanical tear in the ventral dura. Patients with these ventral dural punctures are well-recognized as representing a large segment of patients with $\mathrm{SIH}$ (48\% in this consecutive series). All patients with type 1 are SLEC-P on MR imaging with or without intracranial findings and show a typical ventral hole in the thecal sac on DSM or dynamic CT myelography., 5,11,20 The site of CSF leakage is occasionally seen associated with a calcific "microspur" at the site of a degenerated disc. ${ }^{11,21}$ Unless imaged in a dynamic fashion so that the initial flow of contrast agent is captured as it travels down the spine, the site of leakage will be obscured by mixing of contrast in the intradural and extradural compartments above and below the hole.

All type 2 CSF leaks were SLEC-P as well. A type 2 leak due to a more lateral tear in the dura was a less common cause of SLEC-P CSF leakage in our series. The SLECs associated with a type 2 leak may tend to be positioned more in the neural foramina and are not associated with degenerative disc disease. These leaks are thought to arise from the base of the nerve root relating to a pre-existing thinned or dehiscent area of the dura. ${ }^{11}$ In our experience, these lateral dural dehiscent lesions are more commonly seen in women ( 4 of 4 in our series) and appear to be more amenable to treatment with multiple EBPs and bedrest, possibly due to the nature of the tear and it not being held open by a disc protrusion or substantiated by continued flow as in a CVF. This lesion was seen in 4 of 31 patients (13\%).

The recent discovery of a CVF by Schievink et $\mathrm{al}^{18}$ provided an extraordinary step forward in the care of patients with SIH. We postulate that the dural defect initiating the formation of a CVF occurs more distally along the nerve root sleeve adjacent to an arachnoid granulation or perhaps where the nerve root sleeve is in close proximity to a venous plexus. In this environment, the extravasated fluid is more likely to find its way into a venous channel. We propose that it is in this manner that an embryonic pseudomeningocele, masquerading as a diverticulum, first insinuates into the venous system and heals, aberrantly creating a 1-way CVF, which we categorize as a type 3 CSF leak (Fig 4). This postulation also accounts for these CVFs being commonly but not invariably seen next to what appears to be a diverticulum. Once established, a CVF will not respond to nondirected EBP because it lies well beyond the epidural compartment. Whether it responds to directed regional EBP remains to be better defined. CT-guided focal fibrin patching may have some success in the hands of other authors; however, in our experience, it has not provided a durable cure. Surgical ligation of a CVF as described by Schievink et al, 5,22 in our experience, is the most efficacious treatment of these lesions.

The least common form of CSF leak we encountered in our series we have termed a type 4 leak. This results from a distal nerve root sleeve leak that does not fistulize into the venous system but rather tracks into and dissipates into the adjacent facial planes (Fig 4). A type 4 leak, because of its distal location, will present as a SLEC-N leak and will not be amenable to EBP. Whether this can 
Table 2: Stratification of patients with SIH by type, management, and outcome and demographics

\begin{tabular}{|c|c|c|c|c|c|c|c|c|c|}
\hline & $\begin{array}{c}\text { No. of } \\
\text { Patients }\end{array}$ & M & $\mathbf{F}$ & $\begin{array}{c}\text { Avg } \\
\text { Age (yr) }\end{array}$ & $\begin{array}{l}\text { OP } \\
\text { Avg }\end{array}$ & $\begin{array}{c}\text { OP } \\
\text { Range }\end{array}$ & $\begin{array}{l}\text { Effectively } \\
\text { Treated } \\
\text { with EBP }\end{array}$ & $\begin{array}{l}\text { No. of } \\
\text { Patients } \\
\text { to Surgery }\end{array}$ & Continued Symptoms \\
\hline \multicolumn{10}{|l|}{ SLEC-P } \\
\hline Type 1 & 15 & 8 & 7 & 46 & NA & NA & 4 & 11 & 1 Back pain \\
\hline Type 2 & 4 & 0 & 4 & 31 & NA & NA & 3 & 1 & 1 Mild headache, 1 back pain \\
\hline Not defined & 2 & 2 & 0 & 53 & NA & NA & 2 & 0 & \\
\hline \multicolumn{10}{|l|}{ SLEC-N } \\
\hline Type 3 & 7 & 2 & 5 & 52 & 8.4 & $0-12$ & 0 & 7 & 1 Awaiting surgery \\
\hline Type 4 & 1 & 1 & 0 & 56 & 0 & 0 & 0 & 1 & \\
\hline Not found & 2 & 0 & 2 & 58 & 10 & 10 & 0 & 0 & Continue unchanged \\
\hline
\end{tabular}

Note:-Avg indicates average; OP Avg, average CSF opening pressure; OP Range, the range of CSF opening pressures seen; NA, not applicable; M, male; F, female.

be treated with CT-guided focal fibrin patching remains to be defined.

Our observations regarding the association of SLECs with differing types of CSF leaks is similar to experiences of other authors. Dobrocky et $\mathrm{al}^{11}$ used DSM as well as conventional and dynamic CT myelography to locate the site of leakage in 14 patients. Their study included only patients with SLECs; thus, the authors did not identify any patients with CVFs in their series. Conversely, Schievink et $\mathrm{al}^{5}$ recently reported 14 cases of CVF, none of which were associated with SLEC. Kranz et $\mathrm{al}^{19}$ have reported their success in using dynamic CT myelography and DSM to identify 22 cases of CVF. In that study, the authors also noted that CVFs "often occur without epidural leak." The 7 cases of CVF in our series were all seen in the thoracic region, were not associated with SLEC, were all paravertebral extending laterally from the neural foramen, were inconsistently associated with small diverticula (3 of 7), and, in our experience, were more prevalent on the left (5 of 7). These findings are similar to recently reported experience with CVF. ${ }^{18,19,22}$

The relatively high prevalence of CVF (type 3 leak) in our series presented here is most likely due to the use of lateral decubitus DSM. Lateral DSM optimizes the visualization of the CVF by flooding the lateral gutters of the thecal sac with high-density contrast agent and profiling those gutters with direct frontal highspatial and high-temporal-resolution myelography. Prone DSM is not optimal for detecting these laterally located CVFs and will commonly result in a false-negative DSM. Spinal MR imaging identified all of these patients as SLEC-N; thus, all were initially in the decubitus position for DSM, substantially increasing the yield of the DSM to identify the leak. This same strategy can no doubt be applied to dynamic CT myelography at those centers where that technique is favored over DSM.

Despite the foregoing, there still are a small number of patients who continue to have SIH in whom a CSF leak cannot be localized. There were 2 such patients in this report. Both patients were negative for SLEC, and both continue to show intracranial MR imaging stigmata. We suspect that these patients have a type 3 or perhaps type 4 leak, which our DSMs failed to identify.

A major question in the SIH arena is what to offer the rapidly growing population of patients with headache with brain and spine MR imaging negative for stigmata of CSF leak in whom a possible diagnosis of SIH continues to be considered. Such patients may still harbor a CSF leak despite normal findings on CSF manometry. ${ }^{1,2,23}$ In this report, 3 symptomatic patients positive for SLEC with no intracranial findings under- went DSM (mandated by their SLEC) and were proved to have a leak. Therefore, we must allow the corollary to be true-that is, there must be a percentage of patients negative for SLEC (types 3 and 4) who are leaking slowly and thus not attaining the threshold to display intracranial MR imaging stigmata but are still nonetheless leaking and having symptoms. MR imaging, like CSF manometry, is not an adequate screening test for this disease. Moreover, as awareness of the syndrome of $\mathrm{SIH}$ increases the population of headache patients inferred to have a positional quality to their headache will undoubtedly grow while the low prevalence a true CSF leak in this population will further decline. A better screening test for SIH is needed. It is inefficient to offer exhaustive DSM with its discomfort, mildly invasive nature, radiation dose, and lack of disseminated expertise to this population of patients as the screening test for SIH.

There is clearly a role for EBP in patients positive for SLEC with SIH. Nine of 21 (43\%) of all patients positive for SLEC were treated successfully with EBP (nondirected and directed) and did not require an operation. Determining early which patients positive for SLEC will do well with nondirected-versus-directed EBP and how many times EBP should be attempted before surgical referral is an area for further investigation. Conversely, in this report, $100 \%$ of the 10 patients negative for SLEC failed all forms of EBP. This observation is not surprising given the fact that all patients negative for SLEC were shown to have a leak that starts lateral to and beyond the confines of the spinal canal and thus predisposes an EBP to failure.

Once again, the strategy of using MR imaging to divide patients with SIH into SLEC-P and SLEC-N is prescriptive in that it also appears to foreshadow which patients may benefit from EBP before prone DSM and those who should go on to lateral decubitus DSM without further delay.

The limitations of this current report are obvious in that it represents the experience of essentially 1 neuroradiologist at 1 large neurosurgical referral center. A strong selection bias exists because all patients in this study had MR imaging findings of SIH. Despite an increasing awareness of SIH, the syndrome remains relatively uncommon, resulting in a limited number of neuroradiologists, anesthesiologists, and neurosurgeons skilled in the techniques of diagnosis and treatment of these patients. Dissemination of the technique of DSM as well as dynamic CT myelography, pooling of data across multiple centers, and development of a less invasive screening test for SIH 
would, no doubt, lead to a more efficient diagnosis and effective treatment of these patients.

\section{CONCLUSIONS}

Using spinal MR imaging to dichotomize patients with SIH into SLEC-P and SLEC-N populations accurately determines the nature of their underlying CSF leak (mechanical dural tear versus CVF or nerve root sleeve leak), correctly predicts in whom autologous nondirected and directed EBP may work and in whom it will predictably fail, and finally prescribes the positioning (prone versus decubitus) for subsequent dynamic myelography providing the most efficient pathway to definitive leak localization and subsequent repair. Using this systematic approach, we have been able to identify the exact site of CSF leakage in 27 (87\%) of 31 consecutive patients referred to our institution with MR imaging evidence of SIH.

\section{ACKNOWLEDGMENTS}

The authors acknowledge the contribution of Wendy Gu, a Biomedical Communicator, for her medical illustrations and help with preparation of the figures for this article.

Disclosures: Philip W. Peng—UNRELATED: Other: equipment support from Fujifilm Sonosite Canada.* Christine Lay-UNRELATED: Consultancy: Novartis, Aralez, Eli Lilly and Company, Allergan*; Payment for Lectures Including Service on Speakers Bureaus: Novartis, Aralez. * Money paid to institution.

\section{REFERENCES}

1. Kranz PG, Gray L, Amrhein TJ. Spontaneous intracranial hypotension: 10 myths and misperceptions. Headache 2018;58:948-59 CrossRef Medline

2. Kranz PG, Malinzak MD, Amrhein TJ, et al. Update on the diagnosis and treatment of spontaneous intracranial hypotension. Curr Pain Headache Rep 2017;21:37 CrossRef Medline

3. Schievink WI. Spontaneous spinal cerebrospinal fluid leaks. Cephalalgia 2008;28:1345-56 CrossRef Medline

4. Schievink WI, Schwartz MS, Maya MM, et al. Lack of causal association between spontaneous intracranial hypotension and cranial cerebrospinal fluid leaks. J Neurosurg 2012;116:749-54 CrossRef Medline

5. Schievink WI, Maya MM, Jean-Pierre S, et al. A classification system of spontaneous spinal CSF leaks. Neurology 2016;87:673-79 CrossRef Medline

6. Farb RI, Forghani R, Lee SK, et al. The venous distension sign: a diagnostic sign of intracranial hypotension at MR imaging of the brain. AJNR Am J Neuroradiol 2007;28:1489-93 CrossRef Medline

7. Forghani R, Farb RI. Diagnosis and temporal evolution of signs of intracranial hypotension on MRI of the brain. Neuroradiology 2008; 50:1025-34 CrossRef Medline

8. Fishman RA, Dillon WP. Dural enhancement and cerebral displacement secondary to intracranial hypotension. Neurology 1993;43: 609-11 CrossRef Medline

9. Kranz PG, Amrhein TJ, Choudhury KR, et al. Time-dependent changes in dural enhancement associated with spontaneous intracranial hypotension. AJR Am J Roentgenol 2016;207:1283-87 CrossRef Medline

10. Mokri B. Spontaneous intracranial hypotension. Continuum (Minneap Minn) 2015;21 (4 Headache):1086-108 CrossRef Medline

11. Dobrocky T, Mosimann PJ, Zibold F, et al. Cryptogenic cerebrospinal fluid leaks in spontaneous intracranial hypotension: role of dynamic CT myelography. Radiology 2018;289:766-72 CrossRef Medline

12. Nakagawa N. Changes in spinal MRI findings for epidural fluid collection in the treatment of intracranial hypotension [in Japanese]. No Shinkei Geka 2016;44:925-33 Medline

13. Verdoorn JT, Luetmer PH, Carr CM, et al. Predicting high-flow spinal CSF leaks in spontaneous intracranial hypotension using a spinal MRI-based algorithm: have repeat CT myelograms been reduced? AJNR Am J Neuroradiol 2016;37:185-88 CrossRef Medline

14. Kranz PG, Tanpitukpongse TP, Choudhury KR, et al. Imaging signs in spontaneous intracranial hypotension: prevalence and relationship to CSF pressure. AJNR Am J Neuroradiol 2016;37:1374-78 CrossRef Medline

15. Schievink WI. Spontaneous spinal cerebrospinal fluid leaks and intracranial hypotension. JAMA 2006;295:2286-96 CrossRef Medline

16. Hoxworth JM, Patel AC, Bosch EP, et al. Localization of a rapid CSF leak with digital subtraction myelography. AJNR Am J Neuroradiol 2009;30:516-19 CrossRef Medline

17. Dillon WP. Challenges in the diagnosis and treatment of spontaneous intracranial hypotension. Radiology 2018;289:773-74 CrossRef Medline

18. Schievink WI, Moser FG, Maya MM. CSF-venous fistula in spontaneous intracranial hypotension. Neurology 2014;83:472-73 CrossRef Medline

19. Kranz PG, Amrhein TJ, Gray L. CSF venous fistulas in spontaneous intracranial hypotension: imaging characteristics on dynamic and CT myelography. AJR Am J Roentgenol 2017;209:1360-66 CrossRef Medline

20. Thielen KR, Sillery JC, Morris JM, et al. Ultrafast dynamic computed tomography myelography for the precise identification of highflow cerebrospinal fluid leaks caused by spiculated spinal osteophytes. J Neurosurg Spine 2015;22:324-31 CrossRef Medline

21. Rosebrock RE, Diehn FE, Luetmer PH, et al. Penetrating osseous spicules causing high-flow ventral CSF leaks in the setting of relatively low BMI: a preliminary study. Clin Neuroradiol 2018;28: 539-43 CrossRef Medline

22. Schievink WI, Moser FG, Maya MM, et al. Digital subtraction myelography for the identification of spontaneous spinal CSF-venous fistulas. J Neurosurg Spine 2016;24:960-64 CrossRef Medline

23. Kranz PG, Tanpitukpongse TP, Choudhury KR, et al. How common is normal cerebrospinal fluid pressure in spontaneous intracranial hypotension? Cephalalgia 2016;36:1209-17 CrossRef Medline 